\title{
La disinformazione del paziente è la prima causa della marginalità della DP nel nostro Paese
}

\author{
Flavia Caputo
}

Nefrologia 2 con Dialisi e Trapianto, ARNAS Ospedale Civico Palermo, Palermo

\begin{abstract}
Wrong INFORMATION ON RENAL REPLACEMENT THERAPY IS THE FIRST CAUSE OF MARGINALIZATION OF PD IN ITALY
Abstract. Peritoneal dialysis still shows only a $10 \%$ prevalence rate since the last decades; in fact, the majority of Italian end-stage kidney disease patients are treated with hemodialysis., this impairment on renal replacement therapies' choice is probably due to the wrong type of information provided to patients by physicians that are unable to explain all odds to this kind of patients; besides, some more "unclear" reasons seem to play a role (e.g. social, economical).
\end{abstract}

Key words: Peritoneal dialysis, Extracorporeal dialysis, Information, Nephrologist, Patient

Conflict of interest: None.

Financial support: None.

Accettato: 16 Agosto 2013

Quanti anni sono passati, Marco, dal primo "giro di opinioni” che hai pubblicato sulla tua rivista? Dieci o più; sicuramente, tanti! E cosa è cambiato nel frattempo?

Scrivevamo dieci anni fa che la dialisi peritoneale è una terapia per pazienti un po'speciali, curati da medici e infermieri un po' speciali, cioè una terapia di nicchia che copriva meno del $10 \%$ della popolazione in trattamento. Abbiamo sempre pensato e detto che questo dipendeva da una non completa e non corretta informazione del paziente al momento della scelta del trattamento sostitutivo della funzione renale. Cosa è cambiato, dunque, nel frattempo? Sembrerebbe nulla o poco: al $10 \%$ eravamo e più o meno al $10 \%$ siamo rimasti.

E, tuttavia, io credo che su due punti occorre riflettere:

1 - la dialisi domiciliare in quanto dialisi extracorporea è praticamente scomparsa, mentre la dialisi peritoneale è ancora lì, viva, forse poco vegeta, e questo non è affatto un risultato da poco;

2 - continuiamo a trattare la stragrande maggioranza di quel $90 \%$ che fa dialisi extracorporea in strutture ospedaliere che comportano costi elevati a fronte di trattamenti cronici ambulatoriali: prima o poi qualcuno dovrà riflettere su questo punto e, in un contesto di riorganizzazione e razionalizzazione della spesa sanitaria, si accorgerà che conviene affidare questi pazienti a strutture più agili e meno costose, dislocate sul territorio e fuori dagli ospedali. Non è poi così difficile immaginare che, a quel punto, tutta la materia sarà rivista e, probabilmente, qualcosa accadrà anche per la peritoneale.
In Sicilia, e in Piemonte prima, qualcosa del genere è accaduto: grazie all'intelligenza e alla lungimiranza dell'Assessore Russo, che ha governato la nostra sanità in questi ultimi anni, è stato deliberato un sostegno economico a favore di coloro che, scegliendo un trattamento domiciliare, consentono un risparmio consistente all'Amministrazione regionale e caricano se stessi e, spesso, anche uno o più componenti della famiglia di oneri non indifferenti.

$\mathrm{Ci}$ saremmo aspettati un immediato balzo in avanti della dialisi peritoneale, ma questo, fatta eccezione di poche realtà limitate, non è successo.

I motivi sono tanti e dei più vari: scarsa divulgazione del decreto, pastoie burocratiche che hanno reso difficile l'erogazione del contributo, ma, soprattutto, la persistente incapacità di dare una corretta informazione al paziente sull'argomento "terapia sostitutiva della funzione renale".

E questo perché chi non è in condizione di offrire la terapia domiciliare, sia nel pubblico che nel privato, non è neanche in condizione di dare una corretta informazione, tranne rare eccezioni.

Ho letto quanto hanno scritto i colleghi, o meglio gli amici, di Brescia e la mia riflessione è che tutto il mondo è paese, perché, a fronte di una realtà così positiva come quella Loro, ne esistono altre, magari confinanti, certamente a gestione pubblica, dove si utilizza poco o per nulla la DP quale terapia sostitutiva dell'IRC.

Da noi, a Palermo, registriamo una buona condizione riguardante l'applicazione della DP: le due UO di Nefrologia dell'Ospedale Civico gestiscono, nel loro insieme, più di 100 
pazienti in terapia domiciliare e l'informazione è ormai non più in discussione, è "scontata".

Ma la Sicilia è grande e non è soltanto Palermo: alcune province stentano ancora tanto ad avviare un programma serio di DP.

È vero che il privato continua a dilagare e io non mi sento di sottovalutare questa nostra condizione particolare, ma non mi sento altrettanto di ridurre tutto a questo!

E allora...? Penso che siamo stati vicinissimi a una svolta epocale qui giù in Sicilia e, come spesso succede quando stai per raggiungere una meta "grandiosa", tutto si è bloccato.

E cambiata la classe politica, sono cambiati gli uomini nei posti chiave, è venuta meno, insomma, la determinazione di cambiare e la voglia di far andare le cose nel verso giusto. Per me, per noi e per tutti coloro che ci hanno creduto, l'accaduto potrebbe indurre alla rassegnazione: questa terra è "irredimibile", che vadano tutti a...

Poi, però, ti vengono in mente tante cose del tuo passato che ti aiutano a superare questi momenti e, soprattutto, ti viene da pensare che il cambiamento c'è stato, magari non quello che avresti voluto, ma in me sono certamente cambiati, in questi 10-12 anni, il modo di sentire e vivere il mio lavoro e di trattare la gente malata che sceglie me per essere curata e a cui cerco di dare una buona informazione per fare una buona scelta riguardante il proprio trattamento di dialisi: è mi fermo qui.

\section{Riassunto}

La dialisi peritoneale è ferma da decenni a una prevalenza intorno al $10 \%$ e la maggioranza dei dializzati del nostro Paese viene trattata mediante emodialisi extracorporea in ospedale. La persistente incapacità di dare una corretta informazione al paziente sull'argomento "terapia sostitutiva della funzione renale" e su tutto quello che vi sta "dietro" deve essere ritenuta una delle principali cause di questa sproporzione.

Parole chiave: Dialisi peritoneale, Dialisi extracorporea, Informazione, Nefrologo, Paziente

Dichiarazione di conflitto di interessi: Gli Autori dichiarano di non avere conflitto di interessi.

Contributi economici degli autori: Gli Autori dichiarano di non aver ricevuto sponsorizzazioni economiche per la preparazione dell'articolo.

Indirizzo degli Autori:

Dr.ssa Flavia Caputo

Nefrologia $2^{\circ}$ con Dialisi e Trapianto

ARNAS Ospedale Civico Palermo

Piazza Nicola Leotta 4

90100 Palermo

flavia.caputo@tin.it 